\title{
EVALUATION OF ADVANCED-PRF WITH XENOGRAFT FOR OBLIT- ERATION OF BONE CAVITY IN ANTERIOR MAXILLARY REGION
}

\author{
Tarek S Mohamed*, Elsaeed M Abdellatif**, Wael A Elmohandes***
}

\begin{abstract}
Objective: was to evaluate the clinical outcomes of the use of Advanced-PRF with xenograft for obliteration of bone cavity in anterior maxillary region. Methods: this study included ten patients suffering from a cystic lesion in anterior maxillary area causing bony defect. Clinical examination was made to ensure the cases and to exclude any interfering maters. Radiographic examination was done preoperatively using digital panoramic $\mathrm{x}$-ray and $\mathrm{CT}$ to ensure size will be of a critical size defect. Endodontic treatment for the corresponding teeth has been done before surgery. A-PRF has been prepared as soon before surgery to enucleate the lesion and apicectomy then xenograft block with A-PRF was put into the cavity. Follow up was done to evaluate clinical and radiographic progress. All reading was recorded and analyzed statistically. Results: The study showed an improvement in clinical features and bone density. Conclusion: The application graft with (A-PRF) is very effective in improving the healing process as well as decreasing the postoperative complication.
\end{abstract}

\section{INTRODUCTION}

Bone cavity is a result of abnormal osteoclastic activity caused by a pathological condition such as abscess, cyst and tumor ${ }^{(1)}$. Small sized bone cavity could heal spontaneously without obliteration ${ }^{(3)}$. However, critical sized bone cavity necessitates obliteration of the cavity using graft materials to serve as asscaffold to promote bone healing ${ }^{(4-7)}$.

Many different materials have been used for filling of such critical sized cavity. Among these are autologous bone grafts, allografts, alloplastic materials and xenografts ${ }^{(8-13)}$. Autografts, are grafts transferred from one site to another site within the same individual. But it needs to have another site in patient's body ${ }^{(12,13)}$.

Allografts, The transplant of an organ or tissue from one individual to another of the same species with a different genotype. It accounts for many human transplants, including those from cadaveric, living related, and living unrelated donors. It relatively matches the recipient in constitutional elements and architecture and is theoretically available adequately. The fundamental problems of this grafting material are antigenicity and the potential for disease transmission. Alloplastic materials are easier to get and used. Hydroxyapatite bone cement seems to hold great promise as a grafting alloplastic material. It can be used as a porous ceramic or as a paste/cement bone graft material in the jaw. But it has poor mechanical properties and low rates of osseointegration (14-17). Xenografts are bovine in origin; Bovine bone granules possess better osteoconductive potential than biogas crystals and hydroxyapatite when tested in New Zealand rabbits and undergo slower resorption ${ }^{(12,18)}$. Additionally, enhancement of the regenerative process of human body by utilizing the patient's own blood is a

\footnotetext{
* Dentist at Ministry of Health.

** Professor, Head of Oral and Maxillofacial Surgery Department, Faculty of Dental Medicine, Boys, Cairo, Al-Azhar University.

*** Professor, Department of Oral and Maxillofacial Surgery Faculty of Dental Medicine, Boys, Cairo, Al-Azhar University.
} 
unique concept in dentistry. Post-surgically, blood clots initiate the healing and regeneration of hard and soft tissues, Platelets play a crucial role in hemostasis and wound healing, platelet growth factors are well known source of healing cytokines. Numerous techniques of autologous platelet concentrates have been developed and applied in oral and maxillofacial surgery ${ }^{(19)}$. Platelet-rich plasma (PRP) is an autologous product derived from whole blood through the process of gradient density centrifugation.Autologous PRPhas been shown to be safe and effective in promoting the natural processes of wound healing, soft tissue reconstruction, and bone reconstruction and augmentation (20, ${ }^{21)}$. Moreover, platelet rich fibrin (PRF) has been considered as a biological revolution in dental field. Using PRF is a way to accelerate and enhance the body's natural wound-healing mechanisms. Platelets primarily are involved in wound healing through clot formation and the release of growth factors that initiate and support wound healing. PRF represents a similarity to the natural healing process. Through which it is a cost-effective way to obtain high concentrations of growth factors for tissue healing and regeneration and considered as autologous platelet storage ${ }^{(1,22)}$. Additionally, advanced PRF (A-PRF) is a new concept for cell- based tissue engineering by means of inflammatory cells. The clinical and scientific outcomes are very exciting: more and earlier vascularization, faster soft tissue growth, release of BMPs and more cytokines than classical PRF. Moreover, the use of A-PRF without anticoagulant may produce a liquid form that can be injected. This could fill all the spaces around the xenograft and it coagulates immediately after the injection ${ }^{(19,23)}$. The idea of using xenograft as a scaffold in association with A-PRF in obliteration of bone cavity may be of value in acceleration of bone healing, a factor that initiate the present study.

\section{PATIENT AND METHODS}

Ten patients were participated in this study; they were selected from those attending the Outpatient Clinic of Oral and Maxillofacial Surgery Department, Faculty of Dental Medicine, Al-Azhar University, Cairo Branch. Then each patient was evaluated radiogarphically using digital panorama 1:1 radiograph. CT was done to evaluated size of the lesion. The treatment plan included root canal therapy with respect to the related teeth. An endodontic therapy was done with Obturation of the involved roots.

\section{A-PRF preparation:}

$20 \mathrm{cc}$ of peripheral blood were drawn from the patient and put in vacuum tubes without anticoagulant, the tubes were put in the centrifuge in a balance way at $1500 \mathrm{rpm}$ for 14 minutes. After centrifugation, 3 layers were obtained: (Fig 1) $1^{*}$ at the bottom of tube - erythrocytes (red blood cells). $2^{*}$ in the middle of tube - concentration of white blood cells and platelets. This is A-PRF (thin jelly film). $3^{*}$ A cellular plasma (platelet poor plasma) was concentrated at the top and was collected by syringe.

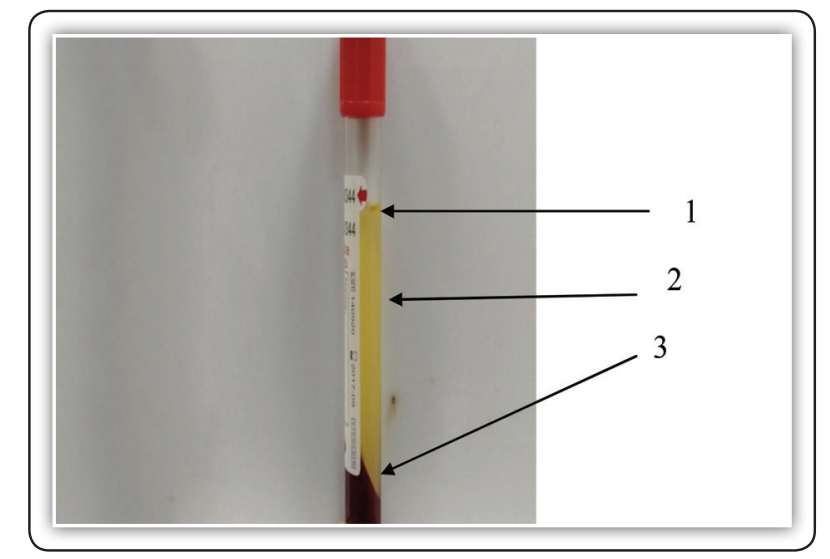

FIG (1) Photograph showing the three layers $(1,2,3)$ obtained after centrifugation.

* Fibrin clots and red corpuscles were removed from the tube with a tweezers; A- PRF clot was immediately separated from red corpuscles by scissor. 


\section{Surgical procedure:}

A trapezoidal full mucoperiosteal flap was made to expose the defected area. First a sulcular incision was made from left canine distal papilla to right lateral incisor distal papilla. Then two releasing incisions making abroad base flap to enhance blood supply to the flap. The flap was reflected carefully not to harm tissues. Loss of cortical plate related to the left maxillary lateral incisor was detected. Osteotomy with surgical burs and irrigation to widen the opening to the defect was made to ensure a better view to the pathos is. Cyst enucleation was done using the convex side of the mucoperiosteal elevator. Apicectomy was done to the root of the lateral incisor with surgical bur and the surgical hand piece under copious irrigation with normal sterile saline. After apicectomy the area was cleaned and irrigated with normal saline to flush any debris and allow good visibility of the site for inspection of any remnant. The osteotomy site was curetted by bone curate to remove the pathos is remnants that might retard the osteointegration. Again, the osteotomy was irrigated by sterile normal saline. (Fig. 2) Xenon graft was delivered to its place in osteotomy cavity. Xenon graft block (EURO-OSS) natural xenon grafting material of coarse $1400 \mu \mathrm{m}$ granules block was $1 * 1 * 2 \mathrm{~cm}^{3}$.

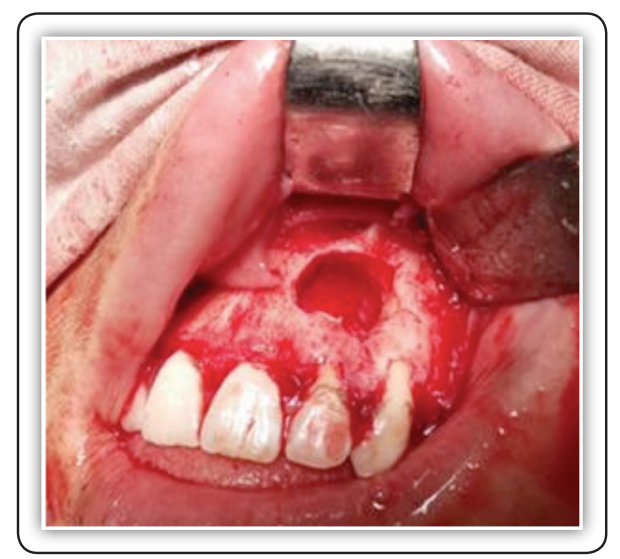

FIG (2) Photograph showing the cavity after curettage.
The pre-prepared A-PRF clot was wedged around the block and the rest was pressed to make a membrane as to cover the grafted bone to stop internal epithelialization. Flap was returned to its place carefully and secured with simple interrupted stitches to the sulcular incision as done to the two releasing ones. Closure was done by 3-0 black silk suture. Medications have been given to each patient after surgery.

\section{Clinical Observations:}

Evaluation of each patient after surgery was performed concerning signs of infection in terms of dehiscence, pain, and swelling. This was done at $1^{\text {st }}$, $3^{\text {rd }}, 5^{\text {th }}, 7^{\text {th }}$ and $14^{\text {th }}$ day after surgery. Stitches were removed one week after surgery. VAS score for pain was calculated. Percent of postoperative swelling change from the preoperative was calculated.

\section{Radiographical assessment:}

The radiographic examination was done with standardized digital panoramic view to assess bone density for each patient preoperatively, immediate postoperative, and at 3 and 6 months postoperatively.

\section{RESULTS}

Dehiscence did not occur in any patient. Hotness occurred in the 1st day in four patients that disappeared on the 2 nd post-operative day in all by using of cold applications. Pain score was 5.7 \pm 1 and ranged from 4.3 to 7 at 1 st day that decrease gradually by time to reach $3.5 \pm 0.6$ in the $3^{\text {rd }}$ day, $2.1 \pm 0.7$ in the $5^{\text {th }}$ day, $1.6 \pm 0.6$ in the $3^{\text {ed }}$ day and finally $0.8 \pm 0.5$ in the $14^{\text {th }}$ day. This was statistically significant $\mathrm{p}<0.001$. Swelling change was $3.6 \% \pm 0.5$ and ranged from 2.9 to 4.3 at $1^{\text {st }}$ day that decrease gradually by time to reach $2.6 \pm 0.6$ in the $3^{\text {rd }}$ day, $1.7 \pm 0.6$ in the $5^{\text {th }}$ day,

$0.9 \pm 0.4$ in the $3^{\text {rd }}$ day and finally $0.2 \pm 0.4$ in the $14^{\text {th }}$ day. This was statistically significant $\mathrm{p}<0.001$. Using the Digora software for Windows, version 1.51, relative bone density, Preoperative bone 
density was54.811 \pm 1.09 and ranged from 53.22 to 56.42 that increase gradually by time to reach $112.705 \pm 1.29$ at the 3rdmonth postoperative, $141.561 \pm 1.25$ in the 6 thmonth. This was statistically significant $\mathrm{p}<0.001$. (Table 1) (Figure 3).

TABLE (1): Mean, SD, median and range of bone density along the clinical follow up periods

\begin{tabular}{|c|c|c|c|c|c|}
\hline BD & Mean & SD & Median & Minimum & Maximum \\
\hline Preoperative & 54.811 & 1.09 & 54.93 & 53.22 & 56.42 \\
\hline 3 months & 112.705 & 1.29 & 112.56 & 110.89 & 114.71 \\
\hline 6 months & 141.561 & 1.25 & 141.375 & 139.61 & 143.31 \\
\hline
\end{tabular}

$P \leq 0.05$ is considered statistically significant, similar letters indicate statistically significant difference.

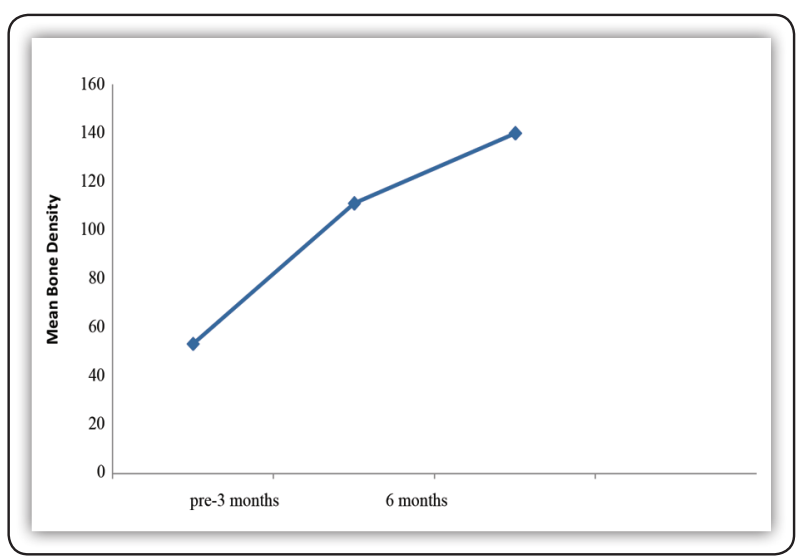

FIG (3) Line diagram represents mean bone density change overtime.

\section{DISCUSSION}

The goal of osseous replacement of these defects are maintenance of contour, elimination of dead space, and reduce postoperative infection, and thus enhance bony and soft tissue healing. Bone grafting is possible because bone tissue has the ability to regenerate completely if provided the space into which has to grow. As natural bone grows, it generally replaces the graft material completely, resulting in a fully integrated region of new bone in addition the main aim of using platelet concentrates specially A-PRF in this thesis stimulate, improve and accelerate healing ${ }^{(24,25)}$. This study used xenograft with A-PRF in filling of maxillary defects, this mixture helped in clot organization, bone growth and decreased post-operative complications. Initial diagnosis of the cases was assisted with the aid of digital panoramic $\mathrm{x}$-ray that gave definite image in diagnosis and accuracy in follow up. X- ray was repeated 3 and 6 months after the surgery to observe bone healing in the site of the surgery. Those patients were free from any systemic or local factors that interfere with the surgical site or with healing process. In this study a trapezoidal full mucoperiosteal flap was made to expose the defect to have a full view and accessibility to the defect and also to provide a good blood supply to the flap. In the current study cyst enucleation, apicectomy, curettage and irrigation all were done to assure removal of all pathological tissue and make it clean cavity. Then xenograft was delivered to the cavity along with the A-PRF then after repositioning with sealed closure of the flap to prevent any dislodgement ${ }^{(22 .}$ ${ }^{23)}$. All healing parameters were positively good and this may be referred to the successful tissue regeneration. This could be explained by the harmonious reaction of various types of cells, including immune response cells (neutrophils, macrophages and lymphocytes), epithelial cells, fibroblasts, stem cells as well as other cells relevant to the tissue. The A-PRF concept seems to be an "ideal" source of components for the healing process (26). Mean values for pain assessment with VAS were high soon after the surgery then gradually decreased this could be due to the increased value of inflammatory process after surgery as result of presence of high concentration of neutrophilic granulocytes that is most commonly considered as early inflammatory cells also bradykinin (which is involved in 
the mechanism of pain) receptors recruit with neutrophils in inflammatory process. Then gradually decreased VAS that may be helped by neutrophilic granulocytes and monocytes/macrophages are in mutual communication. Then they contribute to further differentiation towards a pro- or anti-inflammatory state of the macrophages ${ }^{(27)}$. Infection and/or dehiscence were absent in this study. This could be referred to the help of body immune response cells or the role of (neutrophils and macrophages) found in the fibrin clot, monocytes migrate into the inflamed area after the influx of neutrophils where then become macrophages, also it stimulates B-lymphocytes and antibody secretion and that Blymphocytes were associated with an increased healing process ${ }^{(28)}$. The increase in bone density in the defects along the observation period could be explained either by that xenograft is highly osteoconductive and stand as an efficient scaffold for clot stabilization and further bone deposition or because of the A-PRF which is also osteoconductive and protein component of platelets which has a role in bone healing through stimulation of mesenchymal stem cells to begin osteoblast differentiation and subsequent calcification ${ }^{(29)}$.

\section{CONCLUSION}

The application of xenograft material in critical size defect after cyst enucleation have an important role in healing process. The application of this graft with advanced platelet rich fibrin (A-PRF) is very effective in improving the healing process as well as decreasing the postoperative complication. The use of advanced platelet rich fibrin can accelerate the osseous regeneration. This improvement in bone cavity healing, reduction in pain, and increase in the bone density signifies and highlights the use of A-PRF with grafting material, certainly as a valid method in inducing and accelerating soft and hard tissue regeneration in those critical size bone defects.

\section{REFERENCES}

1. Mazumdar P, Nag D and Bhunia S; Treatment of Periapical Lesion with Platelet Rich Fibrin. Indian Medical Gazette; 2013 ; 4: 28-33

2. Robert J, Gorlind D, Anandp C and Jens P; Odontogenic tumors classification, histopathology, and clinical behavior in man and domesticated animals. American Cancer Society; 1961; 14: 73-101.

3. Vier F and Figueiredo J; Prevalence of different periapical lesions associated with human teeth and their correlation with the presence and extension of apical external root resorption. Int Endod J; 2002; 35: 710-09.

4. Brave D, Madhusudan S, Ramesh G and Brave V; Radicular cyst of anterior maxilla. Int J Dent Clinc; 2011; 3: 16-19.

5. Arx T, Gerber C and Hardt N; Periradicular surgery of molars: a prospective clinical study with a one-year followup. Int Endo J; 2001; 34: 520-005.

6. Manwar N, Agrawal A and Chandak M; Management of infected radicular cyst by surgical approach. Int J Dent Clinc; 2011; 3: 75-09.

7. Brugnami $\mathrm{F}$ and Mellonig J; Treatment of a large periapical lesion with loss of labial cortical plate using GTR. Int J Perio Rest Dent; 1999; 19: 243-009.

8. Caicoya $\mathrm{S}$; Bone cavity filling with alloplastic material in maxillofacial surgery. Rev Esp Cirug Oral y Maxilofac; 2007; 29: 21-32.

9. Alfotawi R and Ayoub A; Reconstruction of maxillofacial bone defects: Contemporary methods and future techniques. Am J Adv Med Sci; 2014; 2:18-27.

10. Vítězslav Z, Jindřich $\mathrm{P}$ and Vladislav $\mathrm{M}$; Bone defects of the facial skeleton - replacement with biomaterials. Biomed; 2003; 147: 51-06.

11. Ochandiano C; Bone cavity filling with alloplastic material in maxillofacial surgery. Rev Esp Cir Oral y Maxilofac; 2007; 29: 21-32.

12. Harry V; Bone Graft Materials. Dent Clin N Am; 2007; 51: 729-046

13. Nandi S, Roy S, Mukherjee P, Kundu B, De D and Basu D; Orthopaedic applications of bone graft \& graft substitutes: a review.Indian J Med Res; 2010; 132:15-30.

14. Gazda A, Lane J, Glaser D and Forster R; Alternatives to Autogenous Bone Graft: Efficacy and Indications. J Am Acad Orthop Sur; 1995; 3: 1-8. 
15. Krishnamurithy G; Potential bone graft substitute for bone tissue engineering application. JUMMEC; 2013; 16: 1-6.

16. Al-Jumaily M, Al-Iraqi M and Al-Naimi K; Biological hydroxyapatite as bone graft substitute; a preliminary report. Ann Coll Med Mosul; 2006; 32: 7-11.

17. Wattanutchariya $\mathrm{W}$ and Changkowchai W; Characterization of porous scaffold from Chitosan-Gelatin/Hydroxyapatite for bone grafting. IMECS; 2014; 2: 12-04.

18. Al Ruhaimi K; Bone graft substitutes: a comparative qualitative histologic review of current osteoconductive grafting materials. Int J Oral Maxillofacial Implants; 2001; 16:105-014.

19. Ghanaati S, Booms P, Orlowska A, Kubesch A, Lorenz J, Rutkowski J, et al; Advanced Platelet-Rich Fibrin (APRF) - A new concept for cell-based tissue engineering by means of inflammatory cells. J Oral Impalntol; 2014; 40: 679- 089 .

20. Ehrenfest D, Andia I, Zumstein M, Zhang C, Pinto N and Bielecki T; Classification of platelet concentrates (Platelet-Rich Plasma-PRP, Platelet-Rich Fibrin-PRF) for topical and infiltrative use in orthopedic and sports medicine: current consensus, clinical implications and perspectives. Muscles Ligaments Tendons J; 2014; 4: 3-9.

21. Smith R, Gassmann C and Campbell M; Platelet-rich Plasma: Properties and Clinical Applications. J Lancaster Gen Hosp; 2007; 2: 73-08.

22. Vijayalakshmi R, Rajmohan C, Deepalakshmi D and Sivakami G; Use of advanced platelet rich fibrin in a fenestration defect around an implant. J Indian Soc Periodontol; 2014; 16: 108-012.

23. Lixin $\mathrm{X}$, yun D, Chaofeng $\mathrm{L}$ and weipeng $\mathrm{J}$; The Use of advance Platelet-Rich Fibrin during immediate implantation for the esthetic replacement of maxillary anterior segment with chronic apical lesion. Int J Sci Res; 2014; 3 : 1368-0072.

24. Friedlaender G; Bone grafts. The basic science rationale for clinical applications. J Bone Joint Surg; 1987; 69A: 786-090.

25. Haile K, Chase S and Herndon C; A comparative study of the healing process following different types of bone transplantation. J Bone Joint Surg; 1963; 45A: 1593-0612.

26. Choukroun J, Ghanaati S, Booms P, Orlowska A, Kubesch A, Lorenz1 J et al; Advanced Platelet-Rich Fibrin (APRF) - A new concept for cell-based tissue engineering by means of inflammatory cells. Journal of Oral Implantology; 2014;40:679-089.

27. Kolaczkowska E and Kubes P; Neutrophil recruitment and function in health and inflammation. Nat Rev Immunol; 2013; 13:159-075.

28. Boyce D, Jones W, Ruge F, Harding K and Moore K; The role of lymphocytes in human dermal wound healing. Br J Dermatol; 2000; 143:59-65.

29. Juan O, Dutan J, Chamberlain T and Croston A; Plateletrich plasma-a adjuvant to wound healing. Int J Perio Res Dent; 2000; 20: 487-095. 